\title{
Who Wants to be IT: An Underappreciated Cause of Lateral Thigh Pain and Paresthesias
}

\author{
Lilian L. Y. Vivas (1), Lawrence R. Robinson (1)
}

Keywords: Electrodiagnostic studies, IT band syndrome, Greater trochanteric pain syndrome, Electromyography doi:10.1017/cjn.2019.336

Can J Neurol Sci. 2020; 47: 253-254

In the childhood game of tag, no-one wants to be "IT". But in the case of lateral thigh numbness and pain presenting to the EMG laboratory, discrete differentials compete for the diagnosis. Much more common than lateral femoral cutaneous neuropathy is Iliotibial band syndrome (ITBS) and its counterpart, greater trochanteric pain syndrome (GTPS). They may be missed in the electromyography (EMG) laboratory, and they represent important musculoskeletal (MSK) mimics for meralgia paresthetica. While there is no evidence of a clinical or electrodiagnostic connection between ITBS/GTPS and meralgia paresthetica, potential confusion between the two and the marked differences in treatment highlight the importance of making the correct diagnosis.

The IT band is a taut fibrous band that is a continuation of the tensor fascia latae (TFL) and attaches to the hip abductors and vastus lateralis. ${ }^{1}$ Distally, it spans to the lateral border of the patella and onto Gerdy's tubercle. ${ }^{1}$ It assists with hip abduction and is an antagonist with TFL for controlling hip adduction during gait. ${ }^{1}$ It slides over the lateral femoral condyle at $30^{\circ}$ of knee flexion and when taut causes friction and irritation. ${ }^{1}$ TFL and hip abductor dysfunction are associated with ITBS. ${ }^{1}$

Simons et al. described radiating patterns of pain and paresthesias of TFL trigger points that extend over the lateral thigh, overlapping with the lateral femoral cutaneous nerve (LFCN) territory. ${ }^{2}$ While the mechanism is unclear, there is an increasing understanding that myofascial pain can cause paresthesia and dysesthesia, thus mimicking a true neurogenic pain generator. ${ }^{3}$ While meralgia paresthetica can present distinctly from ITBS, it may co-exist with other causes of lateral thigh pain and paresthesia, including ITBS. ${ }^{4}$

The IT band also overlies the greater trochanter, which has discrete bursae that serve to cushion tendinous attachments. ${ }^{5}$ Inflammation of the greater trochanteric area elicits pain and often burning, extending along the length of the lateral thigh with highest concentration at the greater trochanter ${ }^{5}$ mimicking the geographic pattern of meralgia paresthetica. GTPS is most commonly a result of tension and/or friction provided by the IT band. ${ }^{5}$

The intricate relationship of ITBS and GTPS should not be separated clinically as they form a "chicken and the egg" scenario. Recent surface EMG data suggest that runners with IT band injury have increased TFL activity after running $3 \mathrm{~min}$ and increased knee adduction after running 30 min. ${ }^{6}$ These findings demonstrate TFL dysfunction and eventual fatigue. This is the catalyst for strain, myofascial soreness and altered kinematics that set the stage for ITBS and GTPS, where trigger point radiation and GTPS pain radiation mimic the pattern of meralgia paresthetica. In non-runners, persons have become more sedentary with fewer lateral movement activities to build hip abductor strength stacked against trends of increasing body mass. Other increasingly common entities like patellofemoral osteoarthritis have been shown to be associated with smaller hip abductor volumes, ${ }^{7}$ correlated with relative weakness and increased likelihood of ITBS and GTPS from dysfunction.

The prevalence of unilateral GTPS in women is $15 \%$ and $6.6 \%$ in men, which is far more common than meralgia paresthetica whose incidence rate is $4.3 / 10,000$ person year ${ }^{7,8}$. At the Sunnybrook Hospital EMG laboratory, we reviewed 1550 records from patients one of the authors (LR) saw over the last 3 years. Of these, 17 patient reports considered "meralgia paresthetica" during the evaluation for lateral thigh pain and/or paresthesias. Only $3 / 17$ of these cases had electrodiagnostically confirmed meralgia paresthetica which has limited diagnostic utility due to LFCN's varied anatomic course. ${ }^{9}$ Eight out of seventeen were given a clinical diagnosis of meralgia paresthetica which is the "gold" standard due to technical limitations of LFCN studies. Importantly, only 1/17 was given a diagnosis of GTPS and also represented the only case where simple MSK tests for GTPS and ITBS were conducted. It is possible, given population data, that a few of the "clinical meralgia paresthetica cases" represented missed diagnosis of the more prevalent GTPS and ITBS. As such, in cases of clinical meralgia paresthetica, it is necessary to also test for GTPS and ITBS which may be missed and contribute to the patient's presentation as an alternate or concurrent diagnosis.

As such, while in the EMG laboratory, the focus is on the peripheral nervous system, there are many surrounding soft tissue structures that can masquerade as neurogenic pain and

From the Department of Physical Medicine and Rehabilitation, University of Toronto, Toronto, ON, Canada

Received July 17, 2019. Final Revisions Submitted November 25, 2019. Date of Acceptance December 4, 2019.

Correspondence to: Lawrence R. Robinson, St. John's Rehab, 285 Cummer Avenue, Room S125, Toronto, ON M2M 2G1, Canada. Email: Larry. Robinson@sunnybrook.ca 
more common clinical entities such as ITBS and GTPS may be the culprits. When assessing patients with lateral thigh paresthesias, it is paramount to palpate for greater trochanteric tenderness, perform modified Thomas test for hip flexor tightness, as well as Ober's and Noble's tests to confront the IT band's tautness. ${ }^{1,10}$ These tests are especially important when sensory examination disturbance is not well demarcated to the exact LFCN distribution, which would remain persistent and well circumscribed in meralgia paresthetica. These tests also do not exclude meralgia paresthetica as a concurrent diagnosis, but aid in diagnosing ITBS/GTPS to help tailor treatment for symptom relief.

Treatment of ITBS and GTPS includes stretching and strengthening the hip abductors, working on optimal kinematics, and treatment of contributory myofascial pain. ${ }^{1,5}$ Topical ice, anti-inflammatories, and corticosteroid injection are some interventions that can aid in the rehabilitative process. ${ }^{10}$ This contrasts with meralgia paresthetica treatment which involves removing restricting clothing, weight loss, nerve blocks, and possible surgical neurolysis. Hence, identifying the correct or even concurrent diagnoses is important for patient outcome.

In summary, when treating patients with lateral thigh pain and paresthesias, diagnoses other than meralgia paresthetica must also be evaluated. The most common musculoskeletal cause for discomfort in this area is IT band syndrome and associated GTPS. Musculoskeletal examination of these structures may improve their detection in the EMG laboratory, leading to more targeted treatment. Common things being common, Mr Occam might be happier with letting the IT band be "it".

\section{CONFLICT OF INTEREST}

We confirm that we have read the Journal's position on issues involved in ethical publication and affirm that this report is consistent with those guidelines. None of the authors have any conflicts of interest to disclose.

\section{Statement of AUTHORShIP}

LV wrote the manuscript, performed the literature search, performed the retrospective chart review, built the reference section, and prepared the manuscript for publication. LR provided editing as well as clinical correlation with his expertise in electromyography. LR was the supervising physician for LV, the resident.

\section{REFERENCES}

1. Fredericson M, Guillet M, DeBenedictis L. Quick solutions for iliotibial band syndrome. Phys Sportsmed. 2000;28(2):52-68.

2. Simons DG, Travell JG, Simons LS. Myofascial pain and dysfunction: the trigger point manual, Volume 2, 2nd ed. Baltimore: Williams and Wilkins; 1998. 281p.

3. Facco E, Ceccherelli F. Myofascial pain mimicking radicular syndromes. Acta Neurochir Suppl. 2005;92:147-150.

4. Rho M, Camacho-Soto A, Cheng A, Havran M, Morone NE, Rodriguez E, Shega J, Weiner DK. Deconstructing chronic low back pain in the older adult - step by step evidence and expert based recommendations for evaluation and treatment. Part VIII: Lateral hip and thigh pain. Pain Med. 2016;17:1249-1260.

5. Williams BS, Cohen SP. Greater trochanteric pain syndrome: a review of anatomy, diagnosis, and treatment. Anesth Analg. 2009;108(5):1662-1670.

6. Baker RL, Souza RB, Rauh MJ, Fredericson M, Rosenthal MD. Differences in knee and hip adduction and hip muscle activation in runners with and without iliotibial band syndrome. PM\&R. 2018;10:1032-1039.

7. Segal NA, Felson DT, Torner JC, Zhu Y, Curtis JR, Niu J, Nevitt MC. Greater trochanteric pain syndrome: epidemiology and associated factors. Arch Phys Med and Rehabil. 2007;88:988-992.

8. Van Slobbe AM, Bohnen AM, Bernsen RM, Koes BW, BiermaZeinstra SM. Incidence rates and determinants in meralgia paresthetica in general practice. J Neurol. 2004;251(3):294-7.

9. Shin YB, Park JH, Kwon DR, Park BK. Variability in conduction of the lateral femoral cutaneous nerve. Muscle Nerve. 2006;33: 645-649.

10. Strauss EJ, Kim S, Calcei JG, Park D. Iliotibial band syndrome: evaluation and management. J Am Acad Orthop Surg. 2011;19:728-736. 